\title{
The Different Effects of BMI and WC on Organ Damage in Patients from a Cardiac Rehabilitation Program after Acute Coronary Syndrome
}

\author{
Lin Xu, ${ }^{1}$ Hui Zhao, ${ }^{1,2}$ Jian Qiu, ${ }^{1}$ Wei Zhu, ${ }^{1}$ Hongqiang Lei, ${ }^{1}$ Zekun Cai, ${ }^{2}$ \\ Wan-Hua Lin, ${ }^{3,4}$ Wenhua Huang, ${ }^{5}$ Heye Zhang, ${ }^{3,4}$ and Yuan-Ting Zhang ${ }^{4,6}$ \\ ${ }^{1}$ Department of Cardiology, Guangzhou General Hospital of Guangzhou Military Region, PLA, Guangzhou, Guangdong 510010, China \\ ${ }^{2}$ Guangzhou University of Chinese Medicine, Guangzhou 510403, China \\ ${ }^{3}$ Institute of Biomedical and Health Engineering, Shenzhen Institutes of Advanced Technology, Shenzhen 518055, China \\ ${ }^{4}$ Key Laboratory for Health Informatics, Chinese Academy of Sciences, Shenzhen 518055, China \\ ${ }^{5}$ Department of Anatomy, Guangdong Provincial Key Laboratory of Medical Biomechanics, School of Basic Medical Science, \\ Southern Medical University, Guangzhou 510515, China \\ ${ }^{6}$ Joint Research Center for Biomedical Engineering, The Chinese University of Hong Kong, Shatin, New Territories 999077, Hong Kong
}

Correspondence should be addressed to Wan-Hua Lin; wh.lin@siat.ac.cn and Wenhua Huang; hwh@fimmu.com

Received 27 November 2014; Accepted 16 February 2015

Academic Editor: Yunmei Chen

Copyright ( $\odot 2015$ Lin Xu et al. This is an open access article distributed under the Creative Commons Attribution License, which permits unrestricted use, distribution, and reproduction in any medium, provided the original work is properly cited.

\begin{abstract}
One of the purposes of cardiac rehabilitation (CR) after acute coronary syndrome (ACS) is to monitor and control weight of the patient. Our study is to compare the different obesity indexes, body mass index (BMI), and waist circumference (WC), through one well-designed CR program (CRP) with ACS in Guangzhou city of Guangdong Province, China, in order to identify different effects of BMI and WC on organ damage. In our work, sixty-one patients between October 2013 and January 2014 fulfilled our study. We collected the vital signs by medical records, the clinical variables of body-metabolic status by fasting blood test, and the organ damage variables by submaximal exercise treadmill test (ETT) and ultrasonic cardiogram (UCG) both on our inpatient and four-to-five weeks of outpatient part of CRP after ACS. We mainly used two-tailed Pearson's test and liner regression to evaluate the relationship of BMI/WC and organ damage. Our results confirmed that WC could be more accurate than BMI to evaluate the cardiac function through the changes of left ventricular structure on the CRP after ACS cases. It makes sense of early diagnosis, valid evaluation, and proper adjustment to ACS in CRP of the obesity individuals in the future.
\end{abstract}

\section{Introduction}

Obesity usually caused a variety of structural adaptations/ alterations that could largely damage cardiovascular structure/function [1]. And cardiac rehabilitation (CR) which is public and well-developed has become a comprehensive management of cardiovascular disease (CVD) clinically [2, 3]. Therefore, one of the purposes of $\mathrm{CR}$ after acute coronary syndrome (ACS) is to monitor and control weight of the patient [4]. Body mass index (BMI) has been a well-accepted index to evaluate the overweight or obese individuals, but it has been reported that BMI fails to reflect true body composition in many studies [5-8]. Gruberg et al. have found the obesity paradox phenomenon, which is the fact that obese patients seemed to survive better in cardiovascular disease populations $[9,10]$. One possible explanation of that is that the popular index of general obesity, BMI, might not be able to accurately reflect the distribution of body fat and fat free mass (FFM) [11-14]. Generally, the increase of body fat is easier to cause CVD than FFM because of metabolic abnormalities [1]. In contrast, waist circumference (WC), a common index to diagnose central obesity, can better reflect body fat than BMI. In other words, WC also has been associated with an increased risk of mortality in patients with CVD [15]; furthermore it might be significant to the prognosis of heart disease. de Koning et al. reported 
a study that consisted of 15,923 subjects with CVD and 5,696 deaths after a median follow-up of 2.3 years. It is said that one centimeter increased in WC was associated with $2 \%$ increase in men and $5 \%$ in women at the risk of future cardiovascular events [16]. Some recent studies explained that high WC could be a potential predictor of endothelial dysfunction, vascular damage and inflammation, and so forth [17-19]. Miyazaki et al. studied 98 patients at 1-2 weeks and at 6 months after ACS, and had founded that the decrease of WC showed to be more related to the progress of endothelial function than the decrease of BMI [17]. Lee et al. reported that it is the WC but not BMI that can reflect the remodeling process after anterior-wall acute myocardial infarction [20]. Moreover, another study also reported that the introduction of CR could have positive effect on BMI, WC, plasma lipoprotein status, and hypertension in obese patients [21]. However, little information is available on the different performance of the BMI and WC indices to associate on the prognosis indexes of CR after ACS, such as the organ damage like the change of cardiovascular structure/function, mortality, and morbidity.

For a better long-term prognosis, we need to monitor and control obesity effectively in CR after ACS. But, before that, we have to confirm the accurate relationship between the severity of obesity and the prognosis. Our study is to compare the different obesity indexes, BMI, and WC, through one well-designed CR program (CRP) on middle-aged and young patients with ACS, in order to identify the prognosis-value of WC in supervising and reducing obesity in the CRP after ACS, especially in the part of the observing organ damage.

\section{Materials and Methods}

2.1. Study Population. The study was conducted in the General Hospital of Guangzhou Military Command of People's Liberation Army (PLA), Guangdong Province, China. Sixtyone patients referred to CRP between October 2013 and January 2014 after percutaneous coronary intervention (PCI) or thrombolytic therapies were recruited into our study. Sixty-one individuals aged $(49.7 \pm 7.9)$ years $(93.3 \%$ males gender) were enrolled in our study. The subjects in this study fulfilled the following inclusion criteria: (1) being aged 1860 years, (2) established ACS, (3) being suitable for the CRP, (4) no weight reduction drug treatment or surgery before participating in this study, and (5) the existence of coronary artery lesions confirmed by coronary angiography (CAG) at the entry. However, the patients with severe complications were eliminated from this study because of potential inconvenience during the CRP, and these severe complications included malignant arrhythmia, acute congestive heart failure decomposition, severe acute pericarditis, myocarditis, systemic inflammatory, cachexy, serious chronic disease, recent thrombosis, or nervous musculoskeletal diseases.

2.2. Measurements. The eligible participant was first directed to finish the medical record that contains the medical information as follows: (1) demographics: age, gender, cardiovascular risk factors including smoking, hypertension, dyslipidemia, and diabetes, and so forth and (2) previous medical history of PCI or thrombolytic therapies, and so forth. Then, vital signs, which were heart rate (HR), blood pressure (BP), diastolic blood pressure (DBP), and weight, were measured after the subject was asked to sit quietly for five minutes. Blood pressure was measured on the left arm. Sixty-one subjects completed this measurement.

2.2.1. The Measurement of BMI, WC. BMI and WC are two measures of obesity in this study. BMI was calculated as weight (in kilograms) divided by the square of height (in meters), and WC was assessed at the midpoint, the narrowest point between the lowest rib and the iliac crest, to the nearest $0.1 \mathrm{~cm}$ using an inflexible tape measure.

We classified the patients into normal group and abnormal group on the basis of BMI and WC, respectively. Abnormal BMI and WC were defined according to the NIH Practical Guide to Obesity and the International Diabetes Federation (IDF) guidelines which were informed by the World Health Organization (WHO) in 2008 [22]. Patients who satisfied the criteria of either overweight or obesity were classified as abnormal group. Specifically, patients with BMI $\geq 25 \mathrm{~kg} / \mathrm{m}^{2}$ were identified as abnormal, and males with WC $>90 \mathrm{~cm}$ or females with WC $>80 \mathrm{~cm}$ were stratified as abnormal [22]. Sixty-one subjects completed this measurement.

2.2.2. Fasting Blood Test. Routine fasting blood test was carried out in the department of clinical biochemistry to collect the blood parameters of fasting blood glucose (FBG, in $\mathrm{mmol} / \mathrm{L}$ ), serum uric acid values (UA, in umol/L), and serum lipids values including total cholesterol (TC, in $\mathrm{mg} / \mathrm{dL}$ ), triglycerides (TG, in $\mathrm{mg} / \mathrm{dL}$ ), low density lipoprotein cholesterol (LDL_C, in $\mathrm{mg} / \mathrm{dL}$ ), and high density lipoprotein cholesterol (HDL_C, in mg/dL). Generally, FBG, serum lipids and UA values represent the metabolic status of our body. The metabolic abnormalities can induce atherosclerosis, thus leading to systematic organ damage [23]. Sixty-one subjects completed this measurement.

2.2.3. Submaximal Exercise Treadmill Test (ETT). Submaximal ETT was carried out according to the Bruce protocol using a sports test machine (CASE, GE Medical Systems: Critikon, Chihuahua). Because of some restrained conditions including the psychological factors and unsuitable symptom, only 21 cases completed the ETT. We documented the parameters as follows: treadmill exercise time (in min), metabolic equivalents (METs), maximum net ST segment deviation (depression or elevation), and Duke treadmill score (DTS). Among them, treadmill exercise time and METs reflect the exercise performance directly and represent the cardiopulmonary function indirectly. Maximum net ST segment deviation and DTS may predict suspected cardiovascular disease (CVD). Twenty-one subjects completed this measurement.

2.2.4. Ultrasonic Cardiogram (UCG). UCG was carried out in the department of echocardiography with an ultrasonic electrocardiogram machine (IE33 S/N, Philips Medical Systems, 
US). We documented the parameters as follows: left ventricular end diastolic dimension (LVDd, $\mathrm{mm}$ ), interventricular septal thickness at diastole (IVSd, mm), left ventricular posterior wall at diastole (LVPWd, mm), ejection fraction (EF, \%), and fractional shortening (FS, \%). Among them, LVDd, IVSd, and LVPWd were three important indices that can reflect the cardiac structure of left ventricular (LV), and $\mathrm{EF}$ and FS were two important indices that can reflect the cardiac ejection function. The enlargement of LV structure and the decline of cardiac ejection function reveal heart lesions. Sixty-one subjects completed this measurement.

2.2.5. Coronary Angiogram (CAG). CAG was performed to detect the lesions of the coronary vascular with a digital subtraction angiography machine (Allura Xper FD20, Philips Medical Systems Nederland B.V.). Left main coronary disease (LMD), three-vessel disease (TVD), and complicated coronary artery disease (CCAD) were diagnosed by doctors based on the results of CAG. PCI was implemented according to the guidance of doctor if the existence of coronary artery lesions were confirmed by the CAG. Sixty-one subjects completed this measurement.

2.2.6. The Procedure of CRP. We designed a specific CRP for the patients participated in this study after the measurements above-mentioned. It consisted of two parts: an inpatientphase part for two-to-three weeks followed by an early outpatient-phase part for four-to-five weeks. The inpatientphase part was for education and counseling of the information of the CRP, risk factor management, nutrition and diet guidance, postoperative activity implementation to the patient, and psychological support, and so forth. The patients were also directed to do appropriate limb movements on a bed and simple walk training for getting out of bed as soon as possible, which could help them to get ready for the early outpatient-phase part. The early outpatient-phase part consisted of a five-minute warm-up and light exercise (stretching), a twenty-minute aerobic exercise (walking or trotting), and a ten-minute cooling-down period (stretching). Each patient carried out the training program three times per week. The exercise intensity was prescribed individually according to $60 \%$ of the maximum intensity at ordinary times or the patient's heart rate (HR) reached approximately $60 \%$ of the maximum $\mathrm{HR}\left(\mathrm{HR}_{\max }\right)$ calculated after ETT. During the training time, the subjective Brog score of patients was about 11-12 points.

2.2.7. Follow-Up. Patients came back to visit us after CRP for four-to-five weeks. Medical records collection, fasting blood test, submaximal ETT, and UCG were performed again according to the measurements above-mentioned. In this period, sixty-one subjects completed the medical records and fasting blood test, thirty-seven subjects completed the ETT, and forty subjects completed the UCG test.

2.3. Statistical Analysis. First of all, baseline clinical characteristics and vital clinical variables of the subjects were studied for normal group and abnormal group, respectively.
Categorical variables were presented as percentages, while continuous variables were present as means \pm SD. The heterogeneity between normal group and abnormal group was assessed by independent-sample $t$ tests and chi-square test. Independent-sample $t$ test was used for the analysis of continuous variables, while chi-square test was for categorical variables. $P$ value $<0.05$ was considered to be significant. Secondly, the correlation of different measures of obesity (i.e., BMI and WC) with vital clinical variables was analyzed using two-tailed Pearson's test. The vital clinical variables in this study included blood pressure, the values of body-metabolic status (i.e., FBG, TC, TG, LDL_C, HDL_C, and UA), variables for the measurement of cardiac structure (i.e., LVDd, IVSd, and LVPWd), and variables for the measurement of cardiac function (i.e., EF, FS, treadmill exercise time, METs, maximum net ST segment deviation, and DTS). Furthermore, a simple linear regression analysis was applied to detect the linear relationship between the measures of obesity and the cardiac structure alteration (i.e., IVSd and LVPWd). After that, multiple linear regression analysis using the backward selection method was performed to estimate the effects of measures of obesity on cardiac structure alteration. WC and BMI were used as independent factors. $P$ value of 0.1 was the criterion for a variable to remain in the model, and $P$ value less than 0.05 was considered statistically significant. Finally, the multiple linear regression analysis was adjusted by age, smoking, hypertension, diabetes, TC, and HDL_C, which were the risk factors used for predicting coronary heart disease in the Framingham Risk Score [24], to verify which measure of obesity was independent of the traditional cardiovascular risk factors. All the analyses were carried out before the CRP and during the follow-up period after CRP twice. The statistical analysis was carried out using SPSS (IBM Company, USA).

\section{Results}

Table 1 shows the baseline clinical characteristics of the sixtyone subjects. Mean \pm SD age was $49.7 \pm 7.9$ years, and 93.3\% were men. Based on BMI standard, 34 and 27 cases were categorized into normal group and abnormal group, respectively. However, based on WC standard, 21 cases and 40 cases were categorized into normal group and abnormal group, respectively. The percentage of subjects with the risk factor of hypertension was significantly higher in the abnormal group than in the normal group regardless of being stratified by BMI or WC standard. The values were $70.4 \%$ versus $26.5 \%(P<0.01)$ and $57.5 \%$ versus $23.8 \%(P<$ $0.05)$ for the two groups that were stratified by BMI and WC standard, respectively. And, there were no significant differences of age, gender, smoking, diabetes, dyslipidemia, left main disease (LMD), three-vessel disease (TVD), and complicated coronary artery disease (CCAD) in the two groups regardless of being stratified by BMI or WC standard.

Table 2 shows the vital clinical variables of the subjects of normal group and abnormal group stratified by BMI and WC standard before CRP. Subjects with abnormal WC 
TABLE 1: Clinical characteristics of the study subjects.

\begin{tabular}{|c|c|c|c|c|c|}
\hline \multirow{2}{*}{ Characteristics } & \multirow{2}{*}{ All subjects $(n=61)$} & \multicolumn{2}{|c|}{ BMI standard } & \multicolumn{2}{|c|}{ WC standard } \\
\hline & & Normal $(n=34)$ & Abnormal $(n=27)$ & Normal $(n=21)$ & Abnormal $(n=40)$ \\
\hline Age (years) & $49.7 \pm 7.9$ & $50.4 \pm 7.7$ & $48.8 \pm 8.1$ & $49.5 \pm 9.2$ & $50.0 \pm 7.2$ \\
\hline Male gender (\%) & $93.3 \%$ & $88.2 \%$ & $96.3 \%$ & $100.0 \%$ & $87.50 \%$ \\
\hline Smoking (\%) & $75.4 \%$ & $73.5 \%$ & $77.8 \%$ & $76.2 \%$ & $75.0 \%$ \\
\hline Weight (kg) & $69.8 \pm 8.9$ & $64.7 \pm 5.8^{* *}$ & $76.3 \pm 7.8^{* *}$ & $64.6 \pm 6.2^{* *}$ & $72.6 \pm 8.9^{* *}$ \\
\hline BMI $\left(\mathrm{kg} / \mathrm{m}^{2}\right)$ & $24.9 \pm 2.6$ & $23.1 \pm 1.4^{* *}$ & $27.2 \pm 1.9^{* *}$ & $23.4 \pm 2.1^{* *}$ & $25.7 \pm 2.5^{* *}$ \\
\hline $\mathrm{WC}(\mathrm{cm})$ & $91.9 \pm 9.6$ & $89.2 \pm 9.3^{* *}$ & $95.4 \pm 8.9^{* *}$ & $82.4 \pm 5.9^{* *}$ & $96.0 \pm 7.0^{* *}$ \\
\hline Diabetes (\%) & $36.1 \%$ & $38.2 \%$ & $33.3 \%$ & $23.8 \%$ & $42.5 \%$ \\
\hline Hypertension (\%) & $45.9 \%$ & $26.5 \%{ }^{* *}$ & $70.4 \%{ }^{* *}$ & $23.8 \%^{*}$ & $57.5 \% *$ \\
\hline Dyslipidemia (\%) & $82 \%$ & $82.4 \%$ & $81.5 \%$ & $71.4 \%$ & $87.5 \%$ \\
\hline $\operatorname{LMD}(\%)$ & $8.2 \%$ & $5.9 \%$ & $11.1 \%$ & $0.0 \%$ & $12.5 \%$ \\
\hline TVD (\%) & $63.9 \%$ & $61.8 \%$ & $66.7 \%$ & $52.4 \%$ & $70.0 \%$ \\
\hline CCAD (\%) & $73.8 \%$ & $76.5 \%$ & $70.4 \%$ & $66.7 \%$ & $77.5 \%$ \\
\hline
\end{tabular}

Notes: ${ }^{*}$ significance of difference is at the 0.05 level. ${ }^{* *}$ Significance of difference is at the 0.01 level.

CRP: cardiac rehabilitation program; BMI: body mass index; WC: waist circumference; LMD: left main disease; TVD: three-vessel disease; CCAD: complicated coronary artery disease.

had significantly higher SBP than those with normal WC $(128.5 \pm 21.9 \mathrm{mmHg}$ versus $120.6 \pm 13.3, P<0.05)$. However, the difference was not significant between the two groups stratified by BMI standard. Beyond that, other vital variables examined by fast blood test, UCG, and ETT appeared as no significant differences between the normal group and the abnormal group regardless of being stratified by BMI or WC standard.

Table 3 depicts the vital clinical variables of the subjects of normal group and abnormal group stratified by BMI and WC standard after CRP. They appeared as several differences in subjects stratified by BMI and WC standard. Firstly, based on BMI standard, 32 and 29 subjects were categorized into normal group and abnormal group, respectively. However, based on WC standard, 24 subjects and 37 subjects were categorized into normal group and abnormal group, respectively. Secondly, subjects with abnormal BMI possessed significantly higher DBP, longer average treadmill exercise time than those with normal BMI. The values were $75.7 \pm 9.6 \mathrm{mmHg}$ versus $68.4 \pm 7.7 \mathrm{mmHg}(P<0.01)$ and $8.9 \pm 2.1 \mathrm{~min}$ versus $7.5 \pm$ $1.6 \min (P<0.05)$, respectively. However, these differences were not significant between the two groups stratified by WC standard. Thirdly, subjects with abnormal WC possess significantly lower HDL_C, higher IVSd, and higher LVPWd than those with normal WC. The values were $41.2 \pm 9.5 \mathrm{mg} / \mathrm{dL}$ versus $47.6 \pm 15.3 \mathrm{mg} / \mathrm{dL}(P<0.05), 9.7 \pm 1.6 \mathrm{~mm}$ versus $8.4 \pm 1.1(P<0.01)$, and $12.3 \pm 2.3 \mathrm{~mm}$ versus $11.0 \pm 1.3 \mathrm{~mm}$ $(P<0.05)$, respectively. However, these differences were not significant between the two groups stratified by BMI standard.

Table 4 depicts Pearson's correlation coefficients of vital clinical variables above-mentioned to BMI and WC before and after CRP. As shown in the table, firstly, BP index (both SBP and DBP) appeared as a positive correlation with BMI both before and after the CRP (SBP before CRP: $r=0.287$, $P=0.025$; SBP after CRP: $r=0.461, P=0.000$; DBP before
CRP: $r=0.279, P=0.029$; DBP after CRP: $r=0.542$, $P=0.000$ ), while the correlation was only significant for WC after CRP (SBP after CRP: $r=0.371, P=0.003$; DBP after CRP: $r=0.309, P=0.015)$. Secondly, the IVSd and LVPWd showed a positive correlation while the HDL_C appeared as a negative correlation with WC but not BMI before CRP and after CRP (IVSd before CRP: $r=0.375, P=0.003$; LVPWd before CRP: $r=0.309, P=0.016$; HDL_C before CRP: $r=-0.256, P=0.046$. IVSd after CRP: $r=0.451, P=0.004$; LVPWd after CRP: $r=0.468, P=0.002$; HDL_C after CRP: $r=-0.292, P=0.022)$. Besides, the FBG, UA, and LDL_C showed a positive correlation with WC but not BMI before CRP (FBG: $r=0.305, P=0.017$; UA: $r=0.369, P=0.003$; LDL_C: $r=0.334, P=0.009)$. Figure 1 shows the different correlation coefficients of BMI and WC to HDL_C, IVSd, and LVPWd before and after CRP.

Table 5 shows the simple linear regression outcomes after CRP. The results showed that WC had a highly linear correlation with indices that reflected cardiac structure alteration (LVSd after CRP: $R^{2}=0.203, P=0.004$; LVPWd after CRP: $\left.R^{2}=0.219, P=0.002\right)$. The correlation scatter diagram was shown in Figure 2. However, BMI had no linear relationship with LVSd and LVPWd $(P>0.05)$.

Table 6 shows the outcomes of multiple linear regression analysis after CRP. In Model 1, LVSd is dependent factor, while WC and BMI as independent factors were entered into the model. The results showed that $\mathrm{WC}$ was the only significant factor (adjusted $R^{2}=0.182, P=0.004$ ) remaining in the model. After adjusted by age, smoking, hypertension, diabetes, TC, and HDL_C, WC still kept in significant correlation with LVSd (Model 2, adjusted $R^{2}=0.305, P=$ 0.001). In Model 3, LVPWd is dependent factor, while WC and BMI as independent factors were entered into the model. The results showed that WC was the only significant factor (adjusted $R^{2}=0.198, P=0.002$ ) remaining in the model. After adjusted by age, smoking, hypertension, diabetes, TC, 
TABLE 2: Vital clinical variables of subjects of the normal group and abnormal group stratified by BMI and WC standards before CRP.

\begin{tabular}{|c|c|c|c|c|c|}
\hline \multirow{2}{*}{ Variables } & \multirow{2}{*}{ All subjects } & \multicolumn{2}{|c|}{ BMI standard } & \multicolumn{2}{|c|}{ WC classified } \\
\hline & & Normal & Abnormal & Normal & Abnormal \\
\hline Smoking (\%) & $75.4 \%(n=61)$ & $73.5 \%(n=34)$ & $77.8 \%(n=27)$ & $76.2 \%(n=21)$ & $75.0 \%(n=40)$ \\
\hline HR (bpm) & $83.2 \pm 15.5(n=61)$ & $83.2 \pm 17.4(n=34)$ & $83.3 \pm 13.0(n=27)$ & $82.4 \pm 17.9(n=21)$ & $83.6 \pm 14.2(n=40)$ \\
\hline $\mathrm{SBP}(\mathrm{mmHg})$ & $128.5 \pm 21.9(n=61)$ & $126.7 \pm 25.1(n=34)$ & $130.8 \pm 17.1(n=27)$ & $120.6 \pm 13.3^{*}(n=21)$ & $128.6 \pm 13.6^{*}(n=40)$ \\
\hline $\mathrm{DBP}(\mathrm{mmHg})$ & $74.5 \pm 12.8(n=61)$ & $72.7 \pm 12.8(n=34)$ & $76.8 \pm 12.6(n=27)$ & $72.3 \pm 11.5(n=21)$ & $75.6 \pm 13.4(n=40)$ \\
\hline $\mathrm{FBG}(\mathrm{mmol} / \mathrm{L})$ & $7.0 \pm 3.0(n=61)$ & $6.8 \pm 2.5(n=34)$ & $7.2 \pm 3.5(n=27)$ & $6.1 \pm 1.9(n=21)$ & $7.4 \pm 3.4(n=40)$ \\
\hline $\mathrm{TC}(\mathrm{mg} / \mathrm{dL})$ & $206.1 \pm 62.2(n=61)$ & $204.2 \pm 59.2(n=34)$ & $208.4 \pm 66.9(n=27)$ & $193.6 \pm 47.3(n=21)$ & $212.6 \pm 68.4(n=40)$ \\
\hline $\mathrm{TG}(\mathrm{mg} / \mathrm{dL})$ & $108.7 \pm 147.1(n=61)$ & $92.2 \pm 140.3(n=34)$ & $129.6 \pm 155.2(n=27)$ & $108.1 \pm 166.7(n=21)$ & $109.1 \pm 137.9(n=40)$ \\
\hline LDL_C (mg/dL) & $123.6 \pm 47.4(n=61)$ & $119.9 \pm 47.7(n=34)$ & $128.3 \pm 47.5(n=27)$ & $110.2 \pm 36.7(n=21)$ & $130.7 \pm 51.1(n=40)$ \\
\hline HDL_C (mg/dL) & $47.4 \pm 13.5(n=61)$ & $49.1 \pm 14.4(n=34)$ & $45.3 \pm 12.3(n=27)$ & $51.8 \pm 16.3(n=21)$ & $45.1 \pm 11.4(n=40)$ \\
\hline $\mathrm{UA}(\mathrm{umol} / \mathrm{L})$ & $368.7 \pm 108.5(n=61)$ & $366.7 \pm 107.5(n=34)$ & $371.1 \pm 111.8(n=27)$ & $337.1 \pm 108.5(n=21)$ & $385.3 \pm 106.1(n=40)$ \\
\hline LVDd (mm) & $51.1 \pm 5.5(n=61)$ & $51.4 \pm 5.3(n=34)$ & $50.6 \pm 5.8(n=27)$ & $50.9 \pm 6.8(n=21)$ & $51.1 \pm 4.7(n=40)$ \\
\hline IVSd (mm) & $9.2 \pm 1.6(n=61)$ & $9.1 \pm 1.7(n=34)$ & $9.3 \pm 1.5(n=27)$ & $8.8 \pm 1.3(n=21)$ & $9.4 \pm 1.7(n=40)$ \\
\hline LVPWd (mm) & $11.7 \pm 1.5(n=61)$ & $11.6 \pm 1.6(n=34)$ & $11.8 \pm 1.5(n=27)$ & $11.3 \pm 1.5(n=21)$ & $11.9 \pm 1.5(n=40)$ \\
\hline $\mathrm{EF}(\%)$ & $58.7 \pm 9.7(n=61)$ & $57.0 \pm 11.0(n=34)$ & $60.7 \pm 7.7(n=27)$ & $57.7 \pm 10.7(n=21)$ & $59.2 \pm 9.3(n=40)$ \\
\hline FS (\%) & $31.8 \pm 8.7(n=61)$ & $31.0 \pm 10.6(n=34)$ & $32.7 \pm 5.3(n=27)$ & $32.0 \pm 12.2(n=21)$ & $31.7 \pm 6.3(n=40)$ \\
\hline $\begin{array}{l}\text { Treadmill exercise } \\
\text { time ( } \min )\end{array}$ & $7.7 \pm 1.7(n=21)$ & $7.7 \pm 1.7(n=11)$ & $7.6 \pm 1.8(n=10)$ & $7.9 \pm 1.9(n=9)$ & $7.5 \pm 1.6(n=12)$ \\
\hline $\begin{array}{l}\text { Metabolic } \\
\text { equivalents }\end{array}$ & $9.4 \pm 2.4(n=21)$ & $9.8 \pm 2.8(n=11)$ & $8.9 \pm 1.8(n=10)$ & $10.0 \pm 3.2(n=9)$ & $8.9 \pm 1.6(n=12)$ \\
\hline $\begin{array}{l}\text { Maximum net ST } \\
\text { segment deviation } \\
(\mathrm{mm})\end{array}$ & $1.1 \pm 1.1(n=21)$ & $1.2 \pm 1.1(n=11)$ & $1.1 \pm 1.2(n=10)$ & $1.3 \pm 1.5(n=9)$ & $1.0 \pm 0.8(n=12)$ \\
\hline DTS & $0.6 \pm 8.2(n=21)$ & $0.8 \pm 8.5(n=11)$ & $0.3 \pm 8.4(n=10)$ & $1.4 \pm 8.3(n=9)$ & $0.0 \pm 8.5(n=12)$ \\
\hline
\end{tabular}

Notes: ${ }^{*}$ significance of difference is at the 0.05 level. ${ }^{* *}$ Significance of difference is at the 0.01 level.

CRP: cardiac rehabilitation program; BMI: body mass index; WC: waist circumference; HR: heart rate; SBP: systolic blood pressure; DBP: diastolic blood pressure; FBG: fasting blood glucose; LDL_C: low density lipoprotein cholesterol; TC: total cholesterol; TG: triglycerides; HDL_C: high density lipoprotein cholesterol; UA: serum uric acid; LVDd: left ventricular and diastolic dimension; IVSd: interventricular septal thickness at diastole; LVPWd: left ventricular posterior wall at diastole; EF: ejection fraction; FS: fractional shortening; DTS: Duke treadmill score.

and HDL_C, WC still kept in significant correlation with LVPWd (Model 4, adjusted $R^{2}=0.269, P=0.002$ ).

\section{Discussions}

This study estimated the different effects of BMI and WC on organ damage in subjects from a CRP after ACS. There were five key findings: (1) obesity assessed by increasing WC was significantly associated with lower HDL_C, higher LVSd, and higher LVPWd in patients from a CRP after ACS. However, the associations were insignificant when the obesity was assessed by BMI. (2) WC had a highly linear correlation with indices that reflected cardiac structure alteration while BMI is not. (3) WC was the only significant factor remaining in the model when a multiple linear regression analysis was performed to estimate the effects of WC and BMI on cardiac structure alteration. (4) After adjusted by age, smoking, hypertension, diabetes, TC, and HDL_C, WC still kept in significant correlation with cardiac structure alteration. (5) Obesity assessed by increasing BMI was significantly associated with higher SBP and DBP in patients from a CRP after ACS. However, when the obesity was assessed by WC, the associations were significant only for the subjects after CRP but not for the subjects before CRP.

4.1. Overweight/Obesity Individuals Assessed by WC and BMI Appeared Different Significant Vital Clinical Variables. Our study shows that the different standard indexes on predicting the degree of obesity may induce the discrepancies of the population characteristics.

Before CRP, the prevalence of hypertension on abnormal BMI/abnormal WC patients is higher than the normal $\mathrm{BMI} /$ normal WC patients (Table 1 ), but $P$ value of the BMI group is larger than WC. And the abnormal WC patients got higher SBP than normal WC patients (Table 2). After CRP, the abnormal BMI patients got higher DBP and shorter treadmill exercise time than normal BMI patients: however, the abnormal WC patients had undergone the lower HDL_C and higher IVSd/LVPWd values than normal WC patients (Tables 2 and 3). Thus, the results show that overweight/obesity individuals assessed by WC and BMI appeared as different vital clinical variables characteristics. Moreover, regardless of being grouped by BMI or WC, overweight/obesity individuals appear as worse parameter of prognosis than the nonobese 
TABLE 3: Vital clinical variables of subjects of the normal group and abnormal group stratified by BMI and WC standards after CRP.

\begin{tabular}{|c|c|c|c|c|c|}
\hline \multirow{2}{*}{ Variables } & \multirow{2}{*}{ All subjects } & \multicolumn{2}{|c|}{ BMI standard } & \multicolumn{2}{|c|}{ WC classified } \\
\hline & & Normal & Abnormal & Normal & Abnormal \\
\hline Smoking (\%) & $18.3 \%(n=61)$ & $15.6 \%(n=32)$ & $20.7 \%(n=29)$ & $16.7 \%(n=24)$ & $18.9 \%(n=37)$ \\
\hline HR (bpm) & $73.8 \pm 11.9(n=61)$ & $74.8 \pm 14.1(n=32)$ & $72.6 \pm 8.8(n=29)$ & $72.6 \pm 15.1(n=24)$ & $75.5 \pm 9.4(n=37)$ \\
\hline $\mathrm{SBP}(\mathrm{mmHg})$ & $124.1 \pm 13.9(n=61)$ & $120.5 \pm 13.9(n=32)$ & $128.1 \pm 13.0(n=29)$ & $120.9 \pm 14.7(n=24)$ & $126.2 \pm 13.2(n=37)$ \\
\hline $\mathrm{DBP}(\mathrm{mmHg})$ & $71.9 \pm 9.3(n=61)$ & $68.4 \pm 7.7^{* *}(n=32)$ & $75.7 \pm 9.6^{* *}(n=29)$ & $69.5 \pm 7.7(n=24)$ & $73.4 \pm 10.0(n=37)$ \\
\hline $\mathrm{FBG}(\mathrm{mmol} / \mathrm{L})$ & $6.4 \pm 2.2(n=61)$ & $6.6 \pm 2.6(n=32)$ & $6.2 \pm 1.7(n=29)$ & $6.4 \pm 2.9(n=24)$ & $6.4 \pm 1.6(n=37)$ \\
\hline $\mathrm{TC}(\mathrm{mg} / \mathrm{dL})$ & $155.7 \pm 43.6(n=61)$ & $158.6 \pm 49.7(n=32)$ & $152.5 \pm 36.4(n=29)$ & $146.4 \pm 29.5(n=24)$ & $161.7 \pm 59.2(n=37)$ \\
\hline $\mathrm{TG}(\mathrm{mg} / \mathrm{dL})$ & $71.6 \pm 54.0(n=61)$ & $68.3 \pm 82.5(n=32)$ & $75.2 \pm 43.6(n=29)$ & $58.1 \pm 34.5(n=24)$ & $80.3 \pm 62.5(n=37)$ \\
\hline LDL_C (mg/dL) & $90.2 \pm 31.2(n=61)$ & $85.7 \pm 24.2(n=32)$ & $95.2 \pm 37.3(n=29)$ & $82.0 \pm 18.5(n=24)$ & $95.5 \pm 36.5(n=37)$ \\
\hline HDL_C (mg/dL) & $43.7 \pm 12.4(n=61)$ & $45.0 \pm 14.3(n=32)$ & $42.3 \pm 9.9(n=29)$ & $47.6 \pm 15.3^{*}(n=24)$ & $41.2 \pm 9.5^{*}(n=37)$ \\
\hline UA (umol/L) & $392.1 \pm 100.5(n=61)$ & $400.1 \pm 107.0(n=32)$ & $384.2 \pm 93.9(n=29)$ & $386.9 \pm 103.1(n=24)$ & $395.5 \pm 100.0(n=37)$ \\
\hline LVDd (mm) & $50.9 \pm 4.5(n=40)$ & $50.9 \pm 4.9(n=22)$ & $50.8 \pm 4.2(n=18)$ & $51.3 \pm 5.2(n=19)$ & $50.5 \pm 3.9(n=21)$ \\
\hline IVSd (mm) & $9.1 \pm 1.5(n=40)$ & $8.9 \pm 1.5(n=22)$ & $9.3 \pm 1.5(n=18)$ & $8.4 \pm \mathbf{1 . 1}^{* *}(n=19)$ & $9.7 \pm 1.6^{* *}(n=21)$ \\
\hline LVPWd (mm) & $11.9 \pm 2.0(n=40)$ & $11.5 \pm 2.0(n=22)$ & $12.3 \pm 2.0(n=18)$ & $11.0 \pm 1.3^{*}(n=19)$ & $12.6 \pm 2.3^{*}(n=21)$ \\
\hline $\mathrm{EF}(\%)$ & $61.9 \pm 8.7(n=40)$ & $61.0 \pm 8.7(n=22)$ & $63.1 \pm 8.8(n=18)$ & $60.7 \pm 9.6(n=19)$ & $63 \pm 7.9(n=21)$ \\
\hline FS (\%) & $33.4 \pm 6.1(n=40)$ & $32.5 \pm 5.7(n=22)$ & $34.6 \pm 6.5(n=18)$ & $32.9 \pm 6.6(n=19)$ & $33.9 \pm 5.7(n=21)$ \\
\hline $\begin{array}{l}\text { Treadmill exercise } \\
\text { time ( } \min )\end{array}$ & $8.16 \pm 1.9(n=37)$ & $8.9 \pm 2.1^{*}(n=17)$ & $7.5 \pm 1.6^{*}(n=20)$ & $8.8 \pm 2.3(n=16)$ & $7.7 \pm 1.5(n=21)$ \\
\hline $\begin{array}{l}\text { Metabolic } \\
\text { equivalents }\end{array}$ & $10.2 \pm 2.6(n=37)$ & $11.0 \pm 2.9(n=17)$ & $9.6 \pm 2.3(n=20)$ & $11.1 \pm 3.4(n=16)$ & $9.5 \pm 1.7(n=21)$ \\
\hline $\begin{array}{l}\text { Maximum net ST } \\
\text { segment deviation } \\
(\mathrm{mm})\end{array}$ & $0.6 \pm 0.8(n=37)$ & $0.6 \pm 0.7(n=17)$ & $0.6 \pm 0.9(n=20)$ & $0.5 \pm 1.0(n=16)$ & $0.6 \pm 0.7(n=21)$ \\
\hline DTS & $5.2 \pm 5.1(n=37)$ & $5.7 \pm 4.7(n=17)$ & $4.8 \pm 5.4(n=20)$ & $6.3 \pm 5.7(n=16)$ & $4.4 \pm 4.5(n=21)$ \\
\hline
\end{tabular}

Notes: ${ }^{*}$ correlation is significant at the 0.05 level. ${ }^{* *}$ Correlation is significant at the 0.01 level.

CRP: cardiac rehabilitation program; BMI: body mass index; WC: waist circumference; HR: heart rate; SBP: systolic blood pressure; DBP: diastolic blood pressure; FBG: fasting blood glucose; LDL_C: low density lipoprotein cholesterol; TC: total cholesterol; TG: triglycerides; HDL_C: high density lipoprotein cholesterol; UA: serum uric acid; LVDd: left ventricular and diastolic dimension; IVSd: interventricular septal thickness at diastole; LVPWd: left ventricular posterior wall at diastole; EF: ejection fraction; FS: fractional shortening; DTS: Duke treadmill score.

individuals. That is mainly because the chronic accumulation of the body fat would lead to the bunch of pathophysiological changes such as the increase of cardiac output, inflammation, metabolic abnormalities, and the atherosclerosis [1]. Such pathophysiological changes play an important role in the organ damage. That is to say, it is consistent with the results of previous researches that the development of organ damage exists in the overweight/obesity individuals.

4.2. Correlation of WC with Cardiac Structure in CRP after ACS. In this study, the finding that obesity assessed by increasing WC was significantly associated with lower HDL_C, higher LVSd, and higher LVPWd in patients from a CRP after ACS mirrors the results of previous researches $[8,25]$. It has proved that overweight and obesity have many adverse effects on hemodynamic and cardiovascular structure and function [8]. The obesity often leads to the increases of filling pressure and volume in the heart, which would increase cardiovascular work, and leads to LV changes with dilation and LV hypertrophy [25]. As we have known, measures of cardiac structure, such as LVDd, IVSd, and
LVPWd, are the most important parameters to early evaluate the size of LV structure which could reflect the cardiac function [26]. It is significant to observe the variation of LV structure in the CRP after ACS, especially on overweight/obesity individuals. BMI and WC are the parameters of obesity, but their functions on evaluating obesity are different to some extent. So the discrepancy between the BMI and $\mathrm{WC}$ indexes on the association of the organ damage also existed.

In our study, no matter before and after the CRP, the IVSd and LVPWd showed a positive correlation while the HDL_C showed a negative correlation with WC. However, BMI could not reflect this phenomenon. Furthermore, linear regression analyses told us that not only the simple but also the multiple linear relationship of WC to IVSd and LVPWd showed the statistically significant correlations (Tables 5, 6 and Figure 2). While WC and BMI especially entered in the same models, only WC was the only significant factor to associate with the IVSd and LVPWd. Because the larger values of IVSd and LVPWd represent the more serious compensatory hypertrophy changes of LV structure. We can 
TABLE 4: The correlation between vital clinical variables and BMI or WC before and after CRP.

\begin{tabular}{|c|c|c|c|c|c|c|c|c|}
\hline \multirow{3}{*}{ Variables } & \multicolumn{4}{|c|}{ BMI } & \multicolumn{4}{|c|}{ WC } \\
\hline & \multicolumn{2}{|c|}{ Before CRP } & \multicolumn{2}{|c|}{ After CRP } & \multicolumn{2}{|c|}{ Before CRP } & \multicolumn{2}{|c|}{ After CRP } \\
\hline & $r$ & $P$ & $r$ & $P$ & $r$ & $P$ & $r$ & $P$ \\
\hline SBP (mmHg) & 0.287 & $0.025^{*}$ & 0.461 & $0.000^{* *}$ & 0.205 & 0.113 & 0.371 & $0.003^{* *}$ \\
\hline DBP (mmHg) & 0.279 & $0.029^{*}$ & 0.542 & $0.000^{* *}$ & 0.184 & 0.157 & 0.309 & $0.015^{*}$ \\
\hline $\mathrm{FBG}(\mathrm{mmol} / \mathrm{L})$ & 0.249 & 0.053 & 0.083 & 0.525 & 0.305 & $0.017^{* *}$ & 0.154 & 0.237 \\
\hline $\mathrm{TC}(\mathrm{mg} / \mathrm{dL})$ & 0.071 & 0.589 & -0.043 & 0.740 & 0.174 & 0.179 & 0.159 & 0.220 \\
\hline TG (mg/dL) & 0.177 & 0.173 & 0.091 & 0.487 & 0.027 & 0.838 & 0.109 & 0.402 \\
\hline LDL_C (mg/dL) & 0.147 & 0.260 & 0.097 & 0.458 & 0.334 & $0.009^{* *}$ & 0.204 & 0.115 \\
\hline HDL_C (mg/dL) & -0.235 & 0.068 & -0.235 & 0.069 & -0.256 & $0.046^{*}$ & -0.292 & $0.022^{*}$ \\
\hline UA (umol/L) & 0.149 & 0.251 & 0.093 & 0.476 & 0.369 & $0.003^{* *}$ & 0.171 & 0.187 \\
\hline LVDd (mm) & -0.023 & 0.863 & -0.095 & 0.559 & 0.017 & 0.899 & 0.044 & 0.786 \\
\hline IVSd (mm) & 0.134 & 0.302 & 0.273 & 0.089 & 0.375 & $0.003^{* *}$ & 0.451 & $\mathbf{0 . 0 0 4}^{* *}$ \\
\hline LVPWd (mm) & 0.179 & 0.166 & 0.271 & 0.091 & 0.309 & $0.016^{*}$ & 0.468 & $0.002^{* *}$ \\
\hline $\mathrm{EF}(\%)$ & 0.128 & 0.324 & 0.182 & 0.262 & -0.101 & 0.439 & 0.036 & 0.824 \\
\hline FS (\%) & 0.072 & 0.582 & 0.225 & 0.163 & -0.086 & 0.508 & 0.029 & 0.861 \\
\hline $\begin{array}{l}\text { Treadmill exercise time } \\
(\mathrm{min})\end{array}$ & -0.048 & 0.837 & -0.269 & 0.107 & -0.129 & 0.579 & -0.164 & 0.333 \\
\hline Metabolic equivalents & -0.247 & 0.279 & -0.267 & 0.110 & -0.213 & 0.353 & -0.211 & 0.210 \\
\hline $\begin{array}{l}\text { Maximum net ST segment } \\
\text { deviation }(\mathrm{mm})\end{array}$ & 0.008 & 0.973 & -0.022 & 0.897 & -0.103 & 0.656 & 0.028 & 0.870 \\
\hline DTS & -0.123 & 0.595 & -0.095 & 0.575 & -0.168 & 0.466 & -0.082 & 0.630 \\
\hline
\end{tabular}

Notes: ${ }^{*}$ correlation is significant at the 0.05 level. ${ }^{* *}$ Correlation is significant at the 0.01 level.

CRP: cardiac rehabilitation program; BMI: body mass index; WC: waist circumference; HR: heart rate; SBP: systolic blood pressure; DBP: diastolic blood pressure; FBG: fasting blood glucose; LDL_C: low density lipoprotein cholesterol; TC: total cholesterol; TG: triglycerides; HDL_C: high density lipoprotein cholesterol; UA: serum uric acid; LVDd: left ventricular and diastolic dimension; IVSd: interventricular septal thickness at diastole; LVPWd: left ventricular posterior wall at diastole; EF: ejection fraction; FS: fractional shortening; DTS: Duke treadmill score.

draw a conclusion that WC is closer associated with the IVSd, LVPWd indexes which represent the cardiac structure than BMI in the CRP after ACS.

A potential mechanism to explain such association is that WC represents central obesity, which can exactly predict the body fat [1]. The increased body fat are easier to cause CVD [27], because of the adipose tissue induce the progress of metabolic disturbance, inflammation state, the neuroendocrine changes, and atherosclerosis including the peripheral vessel and coronary artery, and so forth [28]. Moreover, central obesity will influence the cardiac structure and function through the increased cardiac load caused by hemodynamic disorder and the hypoxia or ischemia on the cardiovascular system [29-31]. All the changes of the cardiovascular system above-mentioned also lead to hypertension, arrhythmia, heart failure, and so forth [29-31]. They mostly reflected the compensatory hypertrophy changes of LV structure (LV remodeling) induced by the increased extra load on the cardiovascular system [29-31]. Furthermore, the results of some studies are consistent with our study. For examples, Turkbey et al. reported a study performed in an obese cohort of 5,098 participants (multiethnic study of atherosclerosis) and showed that higher LV mass-to-volume ratio changing in cardiac structure was linearly correlated with higher adiposity measurements such as the WHR, WC [32]. And Apridonidze et al. reported that increased waist circumference can strongly predict the LV hypertrophy [33]. According to results and analyzes above, WC should favorably be correlated with the cardiac structure on the CRP after ACS.

However, BMI is not significantly associated with the cardiac structure in our study. That is probably because BMI is easy to be affected by the weight of bone and muscle, which cannot truly reflect the body fat [34]. Besides, BMI fails to represent the individual difference about the distribution area of adipose tissue of our body [35]. In other words, BMI cannot distinguish the increased mass of fat and lean, so its information on general obesity could be suspicious. There is limited research on comparing BMI and WC to be associated with cardiac structure directly. Yet, Canepa et al. reported a study that WC and BMI were both associated with LV diastolic dysfunction when they were included in the logistic regression models separately. However, when both WC and BMI were in the same model, only WC remained significantly associated with LV diastolic dysfunction [36]. Bombelli et al. reported that, comparing to BMI, WC was the only index that showed statistically significant association with the trend of LV mass index after 10-year follow-up [37]. That is to say, the correlation of BMI and the cardiac structure or function on the CRP after ACS may not be accurate.

Moreover, the results of our study also indicated that the FBG, UA, and LDL_C indexes presented a positive correlation 


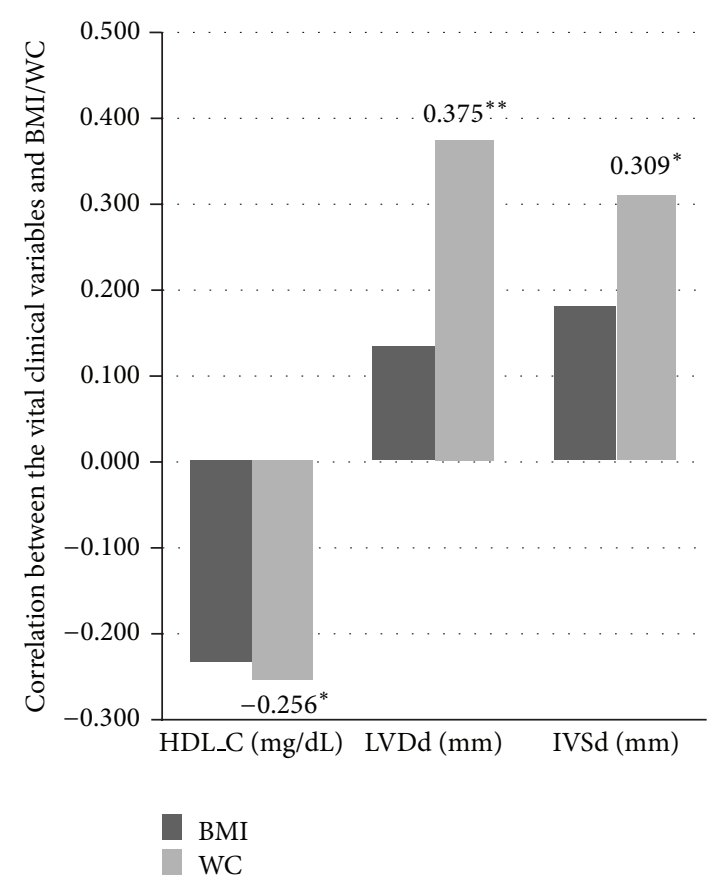

(a) Values of HDL_C, IVSd, and LVPWd before CRP

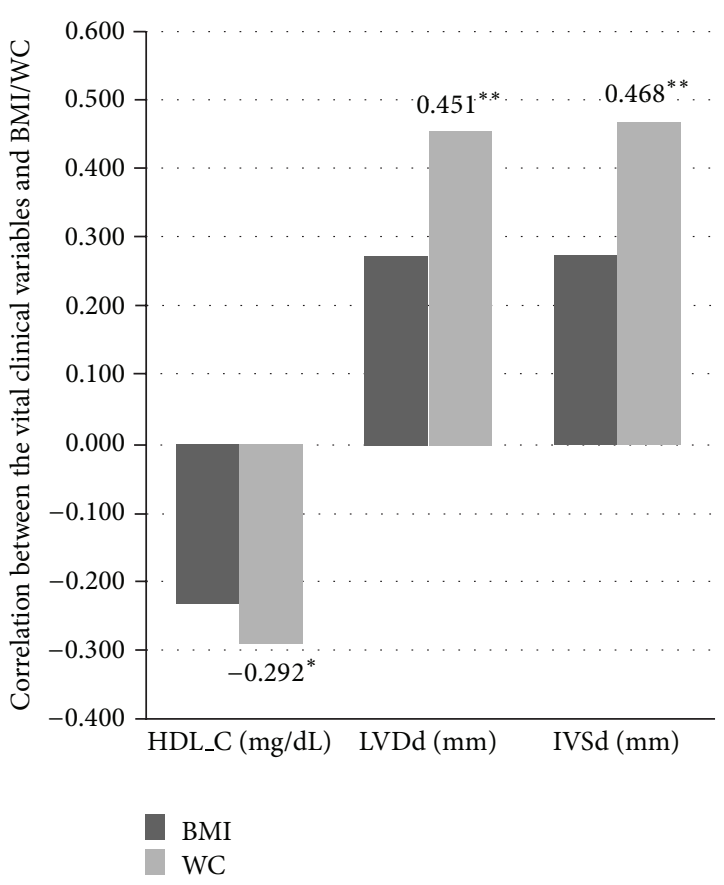

(b) Values of HDL_C, IVSd, and LVPWd after CRP

FIGURE 1: The correlation of the significant variables and BMI/WC in all subjects before and after CRP. Notes: ${ }^{*}$ correlation is significant at the 0.05 level. ${ }^{* *}$ Correlation is significant at the 0.01 level. CRP: cardiac rehabilitation program; BMI: body mass index; WC: waist circumference; SBP: systolic blood pressure; DBP: diastolic blood pressure; IVSd: interventricular septal thickness at diastole; LVPWd: left ventricular posterior wall at diastole.

with WC just before CRP and the HR index showed a positive correlation with WC after CRP, but BMI did not show the correlation before and after CRP (Table 5). The previous researches told us that obesity, hypertension, dyslipidemia, insulin resistance and type 2 diabetes mellitus, and so forth are associated with the prevalence of CVD [1]; they are defined as cardiovascular risk factors. That is to say, WC may be significant to the correlation of the CVD, because of the more meaningful associations of vital clinical indexes than BMI.

Finally, the multiple linear relationship analysis also told us that age and diabetes together with WC have significant correlation with IVSd and the same as age and hypertension, together with WC to LVPWd on the CRP after ACS. In other words, age, hypertensions, and diabetes together with WC also make sense for the cardiac structure on CPR after ACS $[38,39]$. As we know above, hypertension and diabetes are the cardiovascular risk factors and they must be relevant to the CVD and have the correlation with the cardiac function and structure. Besides, aging can cause the decline of the whole body function including the cardiovascular system. That might explain the results above.

4.3. BMI to Correlate with BP in CRP after ACS. According to the BMI standard group on our study, BMI is associated with BP both before and after CRP, and the positive correlation coefficient had mildly improved after CRP no matter SBP or
DBP; the WC standard group also showed us the same positive correlation coefficient after CRP, the BMI standard group manifested more significantly different than WC (Table 5).

As we have known, obesity is associated with hypertension and the mechanisms are multifactorial [40]. At first, obesity can easily lead to dyslipidemia, insulin resistance and hyperinsulinemia, inflammation reaction [40]. Secondly, obesity could induce the endothelial dysfunction and increased vascular stiffness [28]. Thirdly, obesity also leads to the increased cardiac output, heart rate, and so forth which would increase the hemodynamic load to cause hypertension [40]. Fourthly, obesity can induce abnormal kidney function, such as the increased glomerular filtration rate (GFR), primary sodium retention, and activation of the renin-angiotensin system (RAS), and so forth [41]. In conclusion, the mechanisms above-mentioned totally could build up to the progress of atherosclerosis, which can contribute to hypertension. So the obesity indexes such as BMI/WC may be the significant correlation to $\mathrm{BP}$ on the CVD, especially in CRP after ACS. However, BM and WC also appear as different function in the correlation.

The result above-mentioned showed us that BMI could be more strongly associated with BP than WC. Some previous studies were consistent with these results. Oda and Kawai reported a local research and concluded that BMI, but not WC, was independently associated with hypertension in apparently healthy Japanese men and women [42]. Feng et al. also reported a study that BMI is strongly associated with 
TABLE 5: The simple linear correlation between IVSd, LVPWd, and BMI/WC after CRP.

\begin{tabular}{|c|c|c|c|c|c|c|}
\hline Dependent factor & Independent factor & $\beta$ & Lower 95\% CI & Upper 95\% CI & $P$ & $R^{2}$ \\
\hline \multirow{2}{*}{ IVSd } & BMI After CRP & 0.148 & -0.023 & 0.319 & 0.089 & \multirow{2}{*}{0.074} \\
\hline & Constant & 5.432 & 1.175 & 9.688 & 0.014 & \\
\hline \multirow{2}{*}{ IVSd } & WC After CRP & 0.08 & 0.028 & 0.131 & $0.004^{* *}$ & \multirow{2}{*}{0.203} \\
\hline & Constant & 2.079 & -2.502 & 6.659 & 0.364 & \\
\hline \multirow{2}{*}{ LVPWd } & BMI After CRP & 0.2 & -0.034 & 0.433 & 0.091 & \multirow{2}{*}{0.219} \\
\hline & Constant & 6.912 & 1.112 & 12.713 & 0.021 & \\
\hline \multirow{2}{*}{ LVPWd } & WC After CRP & 0.113 & 0.043 & 0.182 & $0.002^{* *}$ & \multirow{2}{*}{0.219} \\
\hline & Constant & 1.945 & -4.23 & 8.12 & 0.528 & \\
\hline
\end{tabular}

Notes: ${ }^{*}$ the simple linear correlation is significant at the 0.05 level. ${ }^{* *}$ Correlation is significant at the 0.01 level.

IVSd: interventricular septal thickness at diastole; LVPWd: left ventricular posterior wall at diastole; BMI: body mass index; WC: waist circumference; CRP: cardiac rehabilitation program; CI: confidence interval.

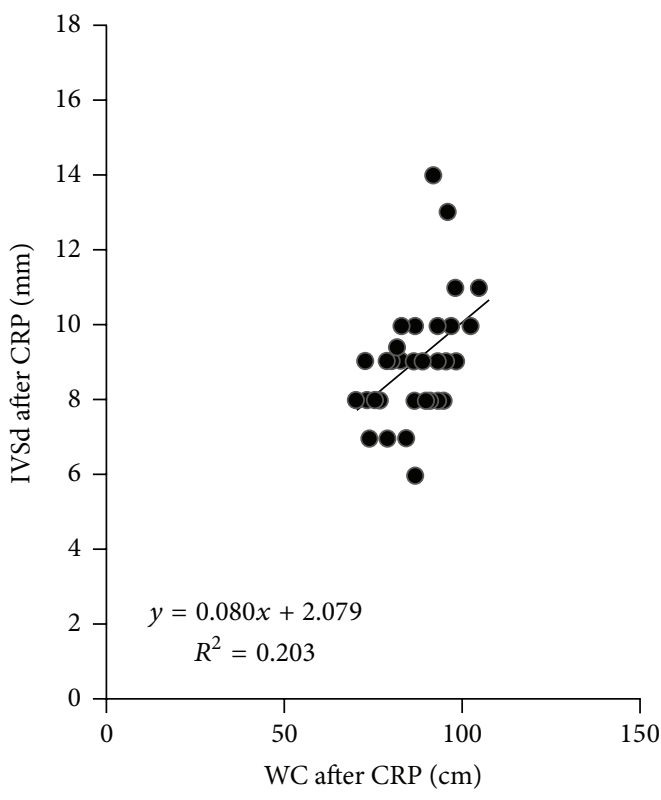

(a)

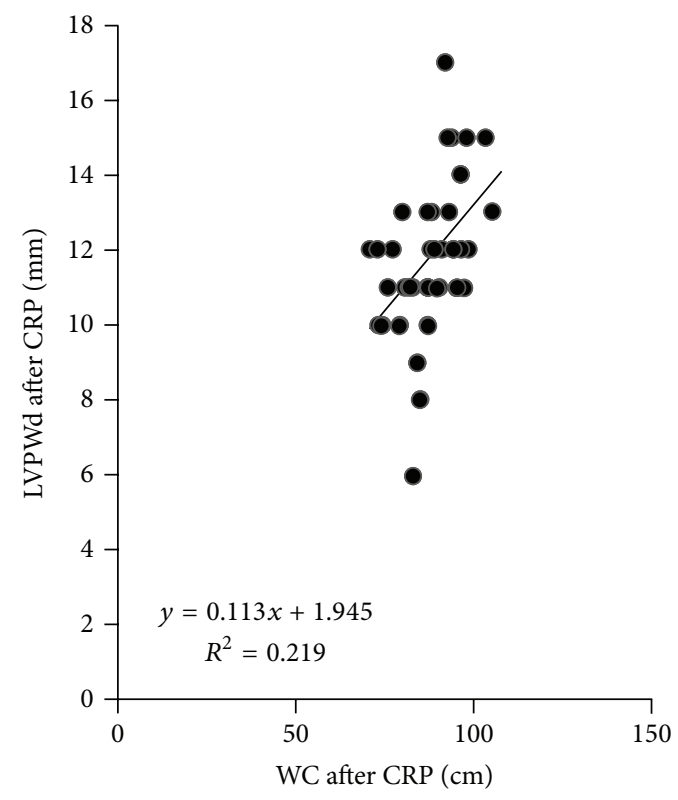

(b)

FIGURE 2: Scatter plots showing the linear correlation between IVSd/LVPWd and WC after CRP. CRP: cardiac rehabilitation program; BMI: body mass index; WC: waist circumference; IVSd: interventricular septal thickness at diastole; LVPWd: left ventricular posterior wall at diastole.

hypertension and WC is strongly associated with diabetes [43]. Blumenthal et al. provided a study and concluded that for overweight or obese persons defined by BMI with above normal BP, exercise and weight loss could produce the larger BP reductions [44]. Mainly reasons are concluded as follows: (1) the Asia population have the larger prevalence on hypertension, when in the same BMI of different ethnic groups; (2) the increased BMI has more directly influence on the body fluid, the vascular resistance, and the heart output which can cause hypertension.

There also some diverse results on different studies, especially in the different gender or ethnic group. Sarno and Monteiro reported a study and concluded that both BMI and abdominal WC were positively and independently associated with the occurrence of arterial hypertension, but the influence of BMI was higher among men [45]. Warren et al. reported a study that abnormal WC was independently associated with a 5-fold risk in hypertension and diabetes in African American women [46]. The explanation of the contradiction is probably due to the different distribution of body fat and FFM between the different gender and ethnicity. Generally, WC which represented central obesity can better reflect body fat than BMI. Furthermore, body fat released lipids into the liver and secreted inflammatory cytokines, which can induce CVD $[43,47]$. So we could infer that WC may also be associated with hypertension.

However, our study presented that BMI is more strongly associated with hypertension than WC and the reasons might be as follows: (1) the gender gap existed in our study (93.3\% males gender), and the population characteristics also showed that overweight/obesity individual assessed by $\mathrm{BMI}$ is more statistically significant with hypertension than WC; (2) it was restricted on the outcomes to use the single BP measurement, and it will be more accurate to use 
TABLE 6: The multiple linear correlation between IVSd, LVPWd, and WC after CRP.

\begin{tabular}{|c|c|c|c|c|c|c|}
\hline Model & $\beta$ & Lower $95 \% \mathrm{CI}$ & Upper 95\% CI & $P$ & Adjusted $R^{2}$ & $P$ \\
\hline \multicolumn{7}{|c|}{ Model 1: dependent factor: IVSd; independent factor: WC, BMI } \\
\hline Constant & 2.079 & -2.502 & 6.659 & 0.364 & \multirow{2}{*}{0.182} & \multirow{2}{*}{$0.004^{* *}$} \\
\hline WC after CRP $(\mathrm{cm})$ & 0.080 & 0.028 & 0.131 & 0.004 & & \\
\hline \multicolumn{7}{|c|}{ Model 2: dependent factor: IVSd; independent factor: WC, age, smoking, hypertension, diabetes, TC, and HDL_C } \\
\hline Constant & -2.847 & -8.294 & 2.599 & 0.296 & \multirow{4}{*}{0.305} & \multirow{4}{*}{$0.001^{* *}$} \\
\hline WC after CRP $(\mathrm{cm})$ & 0.098 & 0.046 & 0.150 & 0.000 & & \\
\hline Age after CRP (y) & 0.073 & 0.021 & 0.124 & 0.007 & & \\
\hline Diabetes after CRP & -0.839 & -1.841 & 0.163 & 0.098 & & \\
\hline \multicolumn{7}{|c|}{ Model 3: dependent factor: LVPWd; independent factor: WC, BMI } \\
\hline Constant & 1.945 & -4.230 & 8.120 & 0.528 & \multirow{2}{*}{0.198} & \multirow{2}{*}{$0.002^{* *}$} \\
\hline WC after CRP $(\mathrm{cm})$ & 0.113 & 0.043 & 0.182 & 0.002 & & \\
\hline \multicolumn{7}{|c|}{ Model 4: dependent factor: LVPWd; independent factor: WC, age, smoking, hypertension, diabetes, TC, and HDL_C } \\
\hline Constant & -3.099 & -10.571 & 4.373 & 0.406 & \multirow{4}{*}{0.269} & \multirow{4}{*}{$0.002^{* *}$} \\
\hline WC after CRP $(\mathrm{cm})$ & 0.140 & 0.069 & 0.212 & 0.000 & & \\
\hline Age after CRP (y) & 0.063 & -0.008 & 0.134 & 0.079 & & \\
\hline Hypertension after CRP & -1.342 & -2.647 & -0.038 & 0.044 & & \\
\hline
\end{tabular}

Notes: ${ }^{*}$ the multiple linear correlation is significant at the 0.05 level. ${ }^{* *}$ Correlation is significant at the 0.01 level.

IVSd: interventricular septal thickness at diastole; LVPWd: left ventricular posterior wall at diastole; WC: waist circumference; CRP: cardiac rehabilitation program; SBP: systolic blood pressure; DBP: diastolic blood pressure; LDL_C: low density lipoprotein cholesterol; CI: confidence interval.

the ambulatory BP measurement to record the BP variability; (3) the limitation of our sample size difference might also cause the outcomes; (4) different studies completed by different ethnic groups might be also considered. In other words, BMI can be associated with BP on the CRP after ACS, but we could not deny the association between WC and BP just judging the result from our study; it needs further studies to confirm their correlation.

4.4. BMI/WC Measurement in Clinical. As we know, WC represents the central obesity in clinical because it can reflect body fat [11-15]. Certain studies have shown that central obesity had a greater risk for CVD than BMI alone [48, 49]. Coutinho et al. reported on a systematic review of the literature and collaborative analysis from about 2,188 studies, suggesting that WC and waist-to-hip ratio (WHR) were more reliable than $\mathrm{BMI}$ in stratifying mortality risk in CVD patients [50]. There are also some studies showing a direct association of WC but not BMI with an increased risk for total mortality $[15,51]$. It means that, to compare with BMI, WC may be more beneficial to evaluate the correlation of organ damage in CVD. It might also be the same performance of the CRP after ACS.

As our study shows the findings above, we can conclude that, to compare with BMI, WC could be more exactly relevant to evaluate the correlation of organ damage on CRP after ACS, especially in the change of LV structure. In other words, we can diagnose that the larger WC patient will get a worse consequence of suffering the heart failure or cardiomegaly and may also be a terrible situation of atherosclerosis progress. That is to say, besides BMI, WC measurement should be considered to focus on our clinical.
For example, WC can be applied to evaluate the degree of obesity for the ACS patients, especially the ones in the CRP. It makes sense of early diagnosis, precise evaluation, and proper treatment to obesity individuals in CRP after ACS in the future clinical care.

4.5. Study Limitations. Nevertheless, our study also manifested shortcomings as follows. First of all, there are 61 cases in our study. The sample size is small, but we follow up all of them about 1 month after CRP. We also compare the data before and after CRP through observing the different effects of WC, BMI on organ damage evaluated by different indexes. Moreover, we also use different and proper statistical analysis methods such as the correlation analysis, simple and multiple linear correlation analysis. We conclude that comparing the BMI measurement, WC makes more sense for the evaluation of organ damage. Secondly, we did not analyze other obesity indexes such as WHR. Thirdly, we did not have the blank control group. Fourthly, we did not consider the influence of medication, diet, and PCI, and so forth. And then, there were not all patients had completed the UCG, EET. Furthermore, we did not follow up a long-term CRP to make more effective evidence. To make up with it, we will try more improved methods in the future.

\section{Conclusions}

Obesity is a risk factor for the development of cardiovascular events. CR is a rewarding progress to intervene in multiple cardiovascular risk profiles [4]. So if we can control obesity effectively via CRP, it means that we can reduce the cardiovascular events. In other words, to understand the accurate 
severity of obesity will make sense to evaluate the correlation of organ damage on CRP after ACS. Our study was about the analysis of the different effects of BMI, WC on the clinical indices including the vital signs, the clinical variables of bodymetabolic status, and the organ damage variables indexes on our designed CRP after ACS. Our result confirmed that WC could be more exactly than BMI to evaluate the cardiac function through the changes of LV structure on the CRP after ACS cases. It makes sense of early diagnosis, valid evaluation, and proper adjustment to ACS in CRP of the obesity individuals in the future.

\section{Ethical Approval}

This study was approved by the Institutional Ethics Committee of the General Hospital of Guangzhou Military Command of PLA.

\section{Consent}

The informed consent was obtained from each subject.

\section{Conflict of Interests}

The authors declare that there is no conflict of interests regarding this paper.

\section{Authors' Contribution}

Lin $\mathrm{Xu}$, Hui Zhao, and Jian Qiu contributed equally to this work.

\section{Acknowledgments}

This work was supported partly by the China Postdoctoral Science Foundation (201003745, 20080440215), the Science and Technology Planning Project of Guangdong Province (2009B011000018, 2013A022100036), the Guangdong Innovation Research Team Fund for Low-Cost Healthcare Technologies in China, the Key Lab for Health Informatics of Chinese Academy of Sciences, and the Shenzhen Innovation Funding (ZDSY20120617113021359).

\section{References}

[1] M. Bastien, P. Poirier, I. Lemieux, and J.-P. Després, “Overview of epidemiology and contribution of obesity to cardiovascular disease," Progress in Cardiovascular Diseases, vol. 56, no. 4, pp. 369-381, 2014.

[2] R. A. Brown, "Rehabilitation of patients with cardiovascular diseases. Report of a WHO Expert Committee," World Health Organization Technical Report Series, vol. 270, pp. 3-46, 1964.

[3] A. S. Leon, B. A. Franklin, F. Costa et al., "Cardiac rehabilitation and secondary prevention of coronary heart disease: an American Heart Association scientific statement from the Council on Clinical Cardiology (Subcommittee on Exercise, Cardiac Rehabilitation, and Prevention) and the Council on Nutrition, Physical Activity, and Metabolism (Subcommittee on Physical Activity), in collaboration with the American association of Cardiovascular and Pulmonary Rehabilitation," Circulation, vol. 111, no. 3, pp. 369-376, 2005.

[4] G. Mckee, M. Kerins, G. Fitzgerald, M. Spain, and K. Morrison, "Factors that influence obesity, functional capacity, anxiety and depression outcomes following a Phase III cardiac rehabilitation programme," Journal of Clinical Nursing, vol. 22, no. 19-20, pp. 2758-2767, 2013.

[5] A. De Schutter, C. J. Lavie, and R. V. Milani, "The impact of obesity on risk factors and prevalence and prognosis of coronary heart disease-the obesity paradox," Progress in Cardiovascular Diseases, vol. 56, no. 4, pp. 401-408, 2014.

[6] P. A. McAuley and K. M. Beavers, "Contribution of cardiorespiratory fitness to the obesity paradox," Progress in Cardiovascular Diseases, vol. 56, no. 4, pp. 434-440, 2014.

[7] R. Arena and L. P. Cahalin, "Evaluation of cardiorespiratory fitness and respiratory muscle function in the obese population," Progress in Cardiovascular Diseases, vol. 56, no. 4, pp. 457-464, 2014.

[8] C. J. Lavie, M. A. Alpert, R. Arena, M. R. Mehra, R. V. Milani, and H. O. Ventura, "Impact of obesity and the obesity paradox on prevalence and prognosis in heart failure," JACC: Heart Failure, vol. 1, no. 2, pp. 93-102, 2013.

[9] L. Gruberg, N. J. Weissman, R. Waksman et al., "The impact of obesity on the short-term and long-term outcomes after percutaneous coronary intervention: the obesity paradox?" Journal of the American College of Cardiology, vol. 39, no. 4, pp. 578-584, 2002.

[10] P. Poirier, "Adiposity and cardiovascular disease: are we using the right definition of obesity?" European Heart Journal, vol. 28, no. 17, pp. 2047-2048, 2007.

[11] C. J. Lavie, R. V. Milani, and H. O. Ventura, "Obesity and cardiovascular disease risk factor, paradox, and impact of weight loss," Journal of the American College of Cardiology, vol. 53, no. 21, pp. 1925-1932, 2009.

[12] C. J. Lavie, A. de Schutter, D. Patel, S. M. Artham, and R. V. Milani, "Body composition and coronary heart disease mortality-an obesity or a lean paradox?" Mayo Clinic Proceedings, vol. 86, no. 9, pp. 857-864, 2011.

[13] C. J. Lavie, R. V. Milani, H. O. Ventura, and A. Romero-Corral, "Body composition and heart failure prevalence and prognosis: getting to the fat of the matter in the 'obesity paradox,"' Mayo Clinic Proceedings, vol. 85, no. 7, pp. 605-608, 2010.

[14] A. Romero-Corral, V. M. Montori, V. K. Somers et al., "Association of bodyweight with total mortality and with cardiovascular events in coronary artery disease: a systematic review of cohort studies," The Lancet, vol. 368, no. 9536, pp. 666-678, 2006.

[15] G. R. Dagenais, Q. L. Yi, J. F. E. Mann, J. Bosch, J. Pogue, and S. Yusuf, "Prognostic impact of body weight and abdominal obesity in women and men with cardiovascular disease," American Heart Journal, vol. 149, no. 1, pp. 54-60, 2005.

[16] L. de Koning, A. T. Merchant, J. Pogue, and S. S. Anand, "Waist circumference and waist-to-hip ratio as predictors of cardiovascular events: meta-regression analysis of prospective studies," European Heart Journal, vol. 28, no. 7, pp. 850-856, 2007.

[17] S.-I. Miyazaki, Y. Hiasa, T. Takahashi et al., "Waist circumference reduction is more strongly correlated with the improvement in endothelial function after acute coronary syndrome than body mass index reduction," Journal of Cardiology, vol. 55, no. 2, pp. 266-273, 2010.

[18] R. Barazzoni, A. Aleksova, C. Carriere et al., "Obesity and high waist circumference are associated with low circulating pen- 
traxin-3 in acute coronary syndrome," Cardiovascular Diabetology, vol. 12, no. 1, article 167, 2013.

[19] C. N. Nunes, M. F. Minicucci, E. Farah et al., "Impact of different obesity assessment methods after acute coronary syndromes," Arquivos Brasileiros de Cardiologia, vol. 103, pp. 19-24, 2014.

[20] M.-J. Lee, Y. Wu, and S. K. Fried, "Adipose tissue heterogeneity: implication of depot differences in adipose tissue for obesity complications," Molecular Aspects of Medicine, vol. 34, no. 1, pp. 1-11, 2013.

[21] P. A. Ades, P. D. Savage, and J. Harvey-Berino, "The treatment of obesity in cardiac rehabilitation," Journal of Cardiopulmonary Rehabilitation and Prevention, vol. 30, no. 5, pp. 289-298, 2010.

[22] WHO Expert Consultation, Waist Circumference and Waist-Hip Ratio, WHO, Geneva, Switzerland, 2008.

[23] P. A. Ades and P. D. Savage, "Potential benefits of weight loss in coronary heart disease," Progress in Cardiovascular Diseases, vol. 56, no. 4, pp. 448-456, 2014.

[24] R. B. D’Agostino Sr., R. S. Vasan, M. J. Pencina et al., "General cardiovascular risk profile for use in primary care: the Framingham heart study," Circulation, vol. 117, no. 6, pp. 743-753, 2008.

[25] D. A. Patel, C. J. Lavie, R. V. Milani, Y. Gilliland, S. Shah, and H. O. Ventura, "Association of left ventricular geometry with left atrial enlargement in patients with preserved ejection fraction," Congestive Heart Failure, vol. 18, no. 1, pp. 4-8, 2012.

[26] H. M. Krumholz, M. Larson, and D. Levy, "Prognosis of leftventricular geometric patterns in the framingham heart-study," Journal of the American College of Cardiology, vol. 25, no. 4, pp. 879-884, 1995.

[27] E. Mourmoura, V. Chaté, K. Couturier et al., "Body adiposity dictates different mechanisms of increased coronary reactivity related to improved in vivo cardiac function," Cardiovascular Diabetology, vol. 13, no. 1, article 54, 2014.

[28] S. L. Henry, B. Barzel, R. J. Wood-Bradley, S. L. Burke, G. A. Head, and J. A. Armitage, "Developmental origins of obesityrelated hypertension," Clinical and Experimental Pharmacology and Physiology, vol. 39, no. 9, pp. 799-806, 2012.

[29] B. Klop, J. W. F. Elte, and M. C. Cabezas, "Dyslipidemia in obesity: mechanisms and potential targets," Nutrients, vol. 5, no. 4, pp. 1218-1240, 2013.

[30] C. J. Lavie, P. A. McAuley, T. S. Church, R. V. Milani, and S. N. Blair, "Obesity and cardiovascular diseases: implications regarding fitness, fatness, and severity in the obesity paradox," Journal of the American College of Cardiology, vol. 63, no. 14, pp. 1345-1354, 2014.

[31] C. J. Lavie, L. P. Cahalin, P. Chase et al., "Impact of cardiorespiratory fitness on the obesity paradox in patients with heart failure," Mayo Clinic Proceedings, vol. 88, no. 3, pp. 251-258, 2013.

[32] E. B. Turkbey, R. L. McClelland, R. A. Kronmal et al., "The impact of obesity on the left ventricle: the Multi-Ethnic Study of Atherosclerosis (MESA)," JACC: Cardiovascular Imaging, vol. 3, no. 3, pp. 266-274, 2010.

[33] T. Apridonidze, H. Shaqra, N. Ktaich, J. E. Liu, and J. N. Bella, "Relation of components of the metabolic syndrome to left ventricular geometry in hispanic and non-hispanic black adults," The American Journal of Cardiovascular Disease, vol. 1, no. 1, pp. 84-91, 2011.

[34] M. Bennasar-Veny, A. A. Lopez-Gonzalez, P. Tauler et al., "Body adiposity index and cardiovascular health risk factors in Caucasians: a comparison with the body mass index and others," PLoS ONE, vol. 8, no. 5, Article ID e63999, 2013.
[35] A. Romero-Corral, V. K. Somers, J. Sierra-Johnson et al., "Diagnostic performance of body mass index to detect obesity in patients with coronary artery disease," European Heart Journal, vol. 28, no. 17, pp. 2087-2093, 2007.

[36] M. Canepa, J. B. Strait, D. Abramov et al., "Contribution of central adiposity to left ventricular diastolic function (from the Baltimore Longitudinal Study of Aging)," The American Journal of Cardiology, vol. 109, no. 8, pp. 1171-1178, 2012.

[37] M. Bombelli, R. Facchetti, R. Sega et al., "Impact of body mass index and waist circumference on the long-term risk of diabetes mellitus, hypertension, and cardiac organ damage," Hypertension, vol. 58, no. 6, pp. 1029-1035, 2011.

[38] G. M. Reaven, "Role of insulin resistance in human disease," Diabetes, vol. 37, no. 12, pp. 1595-1607, 1988.

[39] C. J. Lavie, R. V. Milani, and H. O. Ventura, "Obesity and cardiovascular disease: risk factor, paradox, and impact of weight loss," Journal of the American College of Cardiology, vol. 53, no. 21, pp. 1925-1932, 2009.

[40] J. E. Hall, "Pathophysiology of obesity hypertension," Current Hypertension Reports, vol. 2, no. 2, pp. 139-147, 2000.

[41] V. Kotsis, S. Stabouli, S. Papakatsika, Z. Rizos, and G. Parati, "Mechanisms of obesity-induced hypertension," Hypertension Research, vol. 33, no. 5, pp. 386-393, 2010.

[42] E. Oda and R. Kawai, "Body mass index is more strongly associated with hypertension than waist circumference in apparently healthy Japanese men and women," Acta Diabetologica, vol. 47, no. 4, pp. 309-313, 2010.

[43] R.-N. Feng, C. Zhao, C. Wang, and et al, "BMI is strongly associated with hypertension, and waist circumference is strongly associated with type 2 diabetes and dyslipidemia, in Northern Chinese adults," Journal of Epidemiology, vol. 22, no. 4, pp. 317323, 2012.

[44] J. A. Blumenthal, M. A. Babyak, A. Hinderliter et al., "Effects of the DASH diet alone and in combination with exercise and weight loss on blood pressure and cardiovascular biomarkers in men and women with high blood pressure: the ENCORE study," Archives of Internal Medicine, vol. 170, no. 2, pp. 126-135, 2010.

[45] F. Sarno and C. A. Monteiro, "Relative importance of body mass index and waist circumference for hypertension in adults," Revista de Saude Publica, vol. 41, no. 5, pp. 788-796, 2007.

[46] T. Y. Warren, S. Wilcox, M. Dowda, and M. Baruth, "Independent association of waist circumference with hypertension and diabetes in african american women, South Carolina, 20072009," Preventing Chronic Disease, vol. 9, no. 5, Article ID 110170 , 2012.

[47] S. Flores-Huerta, M. Klünder-Klünder, L. Reyes de la Cruz, and J. I. Santos, "Increase in body mass index and waist circumference is associated with high blood pressure in children and adolescents in Mexico city," Archives of Medical Research, vol. 40, no. 3, pp. 208-215, 2009.

[48] S. D. Hsieh and H. Yoshinaga, "Abdominal fat distribution and coronary heart disease risk factors in men-waist/height ratio as a simple and useful predictor," International Journal of Obesity, vol. 19, no. 8, pp. 585-589, 1995.

[49] E. B. Rimm, M. J. Stampfer, E. Giovannucci et al., "Body-size and fat distribution as predictors of coronary heart-disease among middle-aged and older US men," The American Journal of Epidemiology, vol. 141, no. 12, pp. 1117-1127, 1995.

[50] T. Coutinho, K. Goel, D. Corrêa De S et al., "Central obesity and survival in subjects with coronary artery disease: a systematic 
review of the literature and collaborative analysis with individual subject data," Journal of the American College of Cardiology, vol. 57, no. 19, pp. 1877-1886, 2011.

[51] C. Kragelund, C. Hassager, P. Hildebrandt, C. Torp-Pedersen, and L. Køber, "Impact of obesity on long-term prognosis following acute myocardial infarction," International Journal of Cardiology, vol. 98, no. 1, pp. 123-131, 2005. 


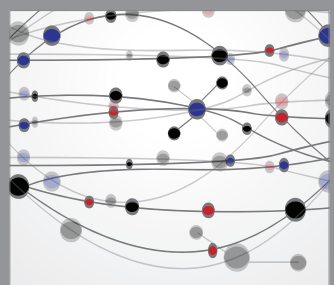

The Scientific World Journal
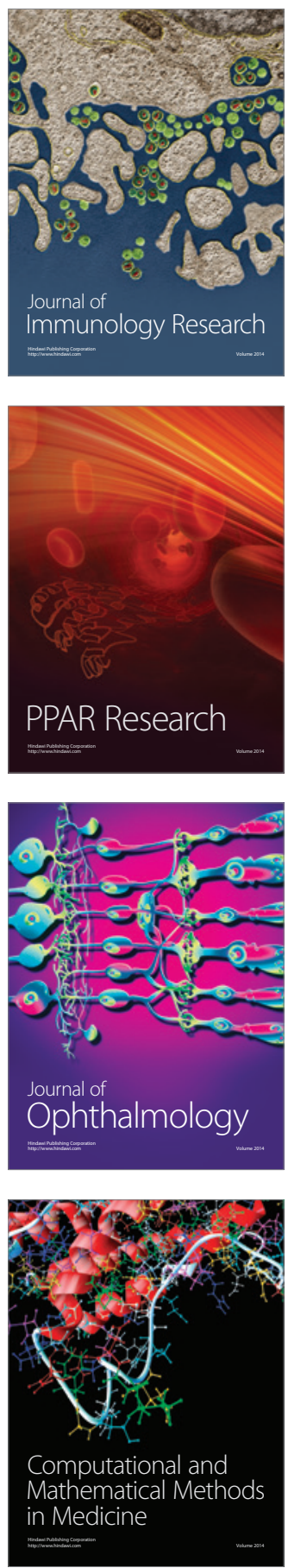

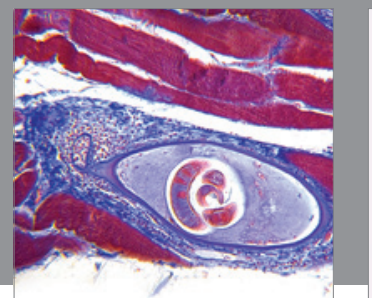

Gastroenterology

Research and Practice
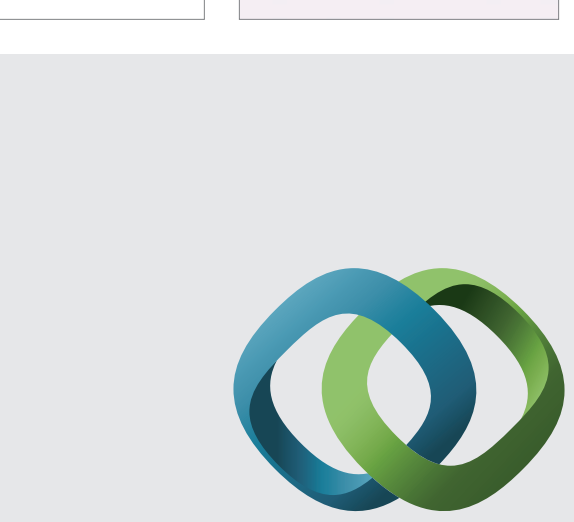

\section{Hindawi}

Submit your manuscripts at

http://www.hindawi.com
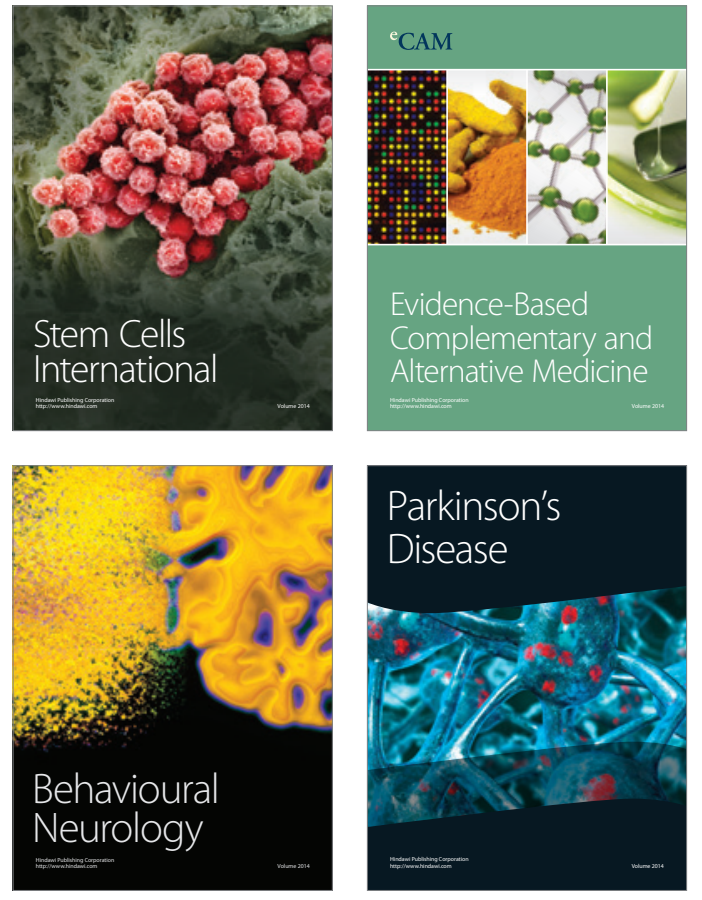
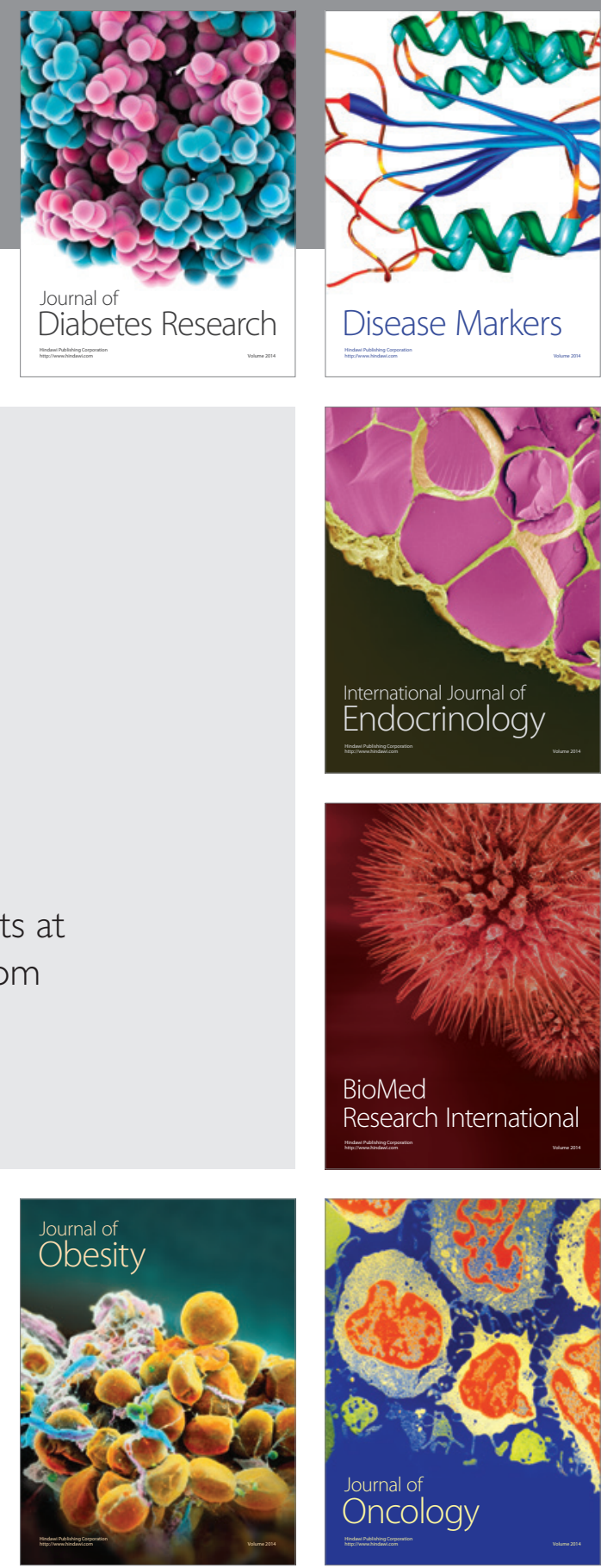

Disease Markers
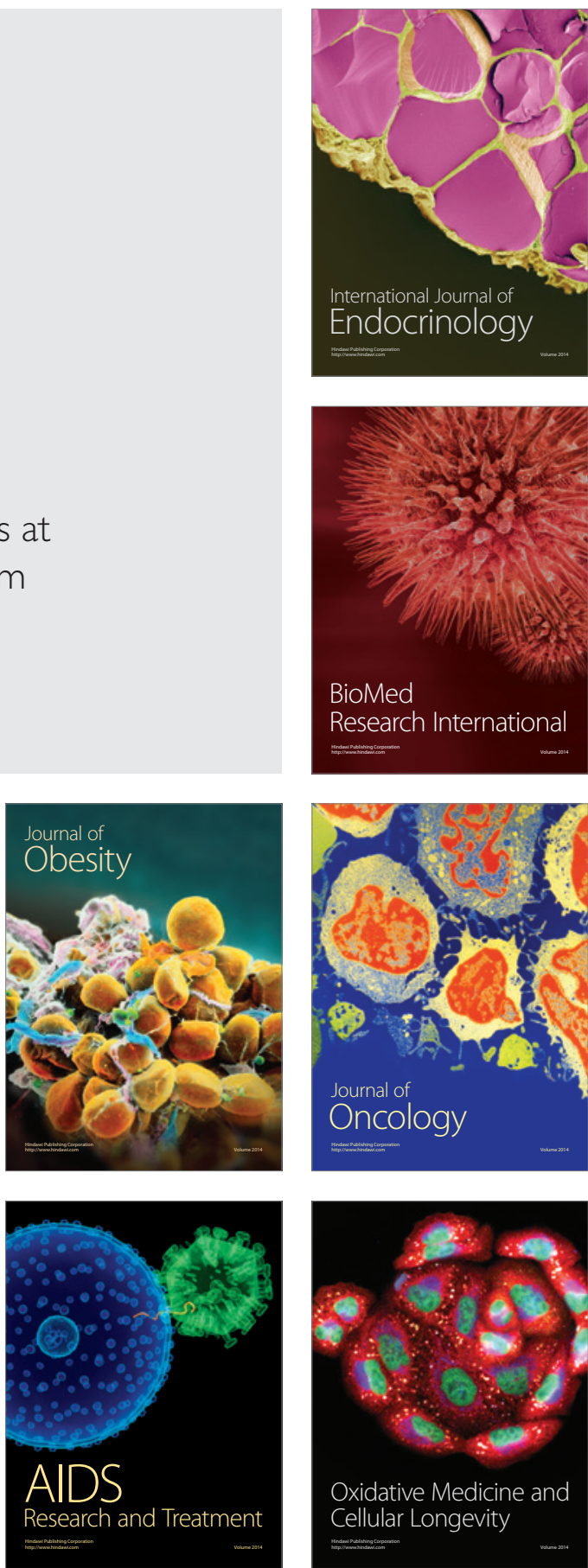